\title{
Serum free testosterone and asthma, asthma hospitalisations and lung function in British adults
}

\author{
Yueh-Ying Han, Qi Yan, Ge Yang, Wei Chen, Erick Forno ํㅣ , Juan Carlos Celedon
}

\begin{abstract}
- Additional material is published online only. To view please visit the journal online (http://dx.doi.org/10.1136/ thoraxjnl-2020-214875)
\end{abstract}

Division of Pediatric Pulmonary Medicine, UPMC Children's Hospital of Pittsburgh, University of Pittsburgh, Pittsburgh, Pennsylvania, USA

\section{Correspondence to}

Dr Juan Carlos Celedon, Pediatric Pulmonology, University of Pittsburgh, Pittsburgh, PA 15224, USA; juan.celedon@chp.edu

EF and JCC are joint senior authors.

Received 23 March 2020 Revised 12 June 2020 Accepted 29 June 2020 Published Online First 31 August 2020

\section{Linked}

http://dx.doi.org/10.1136/ thoraxjnl-2020-215566

Check for updates

(C) Author(s) (or their employer(s)) 2020. No commercial re-use. See rights and permissions. Published by BMJ.

To cite: Han Y-Y, Yan Q, Yang $\mathrm{G}$, et al. Thorax 2020;75:849-854.

\section{ABSTRACT}

Objective We examined the relation between

serum-free testosterone and asthma, wheeze, asthma hospitalisations and lung function in older adults.

Design Cross-sectional study.

Setting UK.

Participants 256419 adults aged 40 to 69 years, recruited from 2006 to 2010.

Main outcome measures Multivariable logistic or linear regression was used for the analysis of free testosterone and physician-diagnosed asthma, current wheeze, asthma hospitalisations and lung function measures, which was adjusted for serum estradiol, smoking status and other covariates.

Results Free testosterone levels above the lowest quartile (Q1) were significantly associated with lower odds of asthma in both women (adjusted OR (aOR) for Q4 (the highest quartile) versus $\mathrm{Q} 1=0.67,95 \% \mathrm{Cl}=0.64$ to 0.71 ) and men (aOR for Q4 versus Q1 $=0.87,95 \% \mathrm{Cl}=0.82$ to 0.91 ). Among subjects with asthma, free testosterone levels above Q1 were significantly associated with lower odds of current wheeze in women (aOR range $=0.78$ to 0.87), and free testosterone levels in Q4 were associated with lower odds of current wheeze in men (aOR for Q4 versus $\mathrm{Q} 1=0.86,95 \% \mathrm{Cl}=0.77$ to 0.95 ). Among women with asthma, free testosterone levels in Q4 were also associated with lower odds of $\geq 1$ asthma hospitalisation. Among men, free testosterone was positively associated with FEV, and FVC. Among women, free testosterone was negatively and weakly associated with FVC.

Conclusion In a large study of British adults, elevated free testosterone levels are associated with lower odds of asthma and current wheeze in women and men, lower odds of asthma hospitalisations in women, and higher FEV, and FVC in men.

Dissemination to participants, and related patient and public communities The results of the study will be linked to the UK Biobank website.

\section{INTRODUCTION}

Asthma affects approximately 339 million people worldwide. ${ }^{1}$ In the UK, asthma leads to 933000 hospitalisations per year, and 1.1 million children and 4.3 million adults are currently receiving treatment for asthma. ${ }^{23}$

The incidence of asthma varies widely by age and sex throughout the lifespan. Although asthma is more common in boys than in girls, adult women have higher prevalence and morbidity from asthma than adult men. ${ }^{4}$ Changes in sex hormone levels during the life course may partly explain this disparity. ${ }^{56}$

\section{Key messages}

What is the key question?

- Are elevated serum levels of free testosterone associated with lower risk of asthma and higher lung function in adults of both sexes?

What is the bottom line?

- In a large population-based study of British adults aged 40 to 69 years, an elevated serum level of free testosterone was associated not only with lower odds of physician-diagnosed asthma and current wheeze in women and men, but also with lower odds of at least one asthma hospitalisation in women and higher $\mathrm{FEV}_{1}$ and $\mathrm{FVC}$ in men.

Why read on?

- The findings from this study suggest that serum-free testosterone is linked to lower risk of asthma in both men and women.

While oestrogen and progesterone may enhance T-helper cell type 2 (Th2)-induced allergic airway inflammation, androgens such as testosterone and 5-alpha dihydrotestosterone $(5 \alpha-\mathrm{DHT})$ may reduce such inflammation by suppressing innate and adaptive immune responses. ${ }^{7}$ Whereas fluctuation in oestrogen or progesterone levels-related to the menstrual cycle, pregnancy, menopause or intake of exogenous hormones-has been linked to increased asthma risk in women, ${ }^{8}$ findings from experimental studies and a small observational study in children suggest that testosterone may be associated with reduced asthma risk in adult men. ${ }^{89}$ In a study of 7615 adults aged 18 to 79 years in the USA, we previously reported that a high serum testosterone level was associated with lower odds of current asthma in women $(n=3662)$ but not in men $(n=3953)$, a finding that could be explained by limited statistical power for the analysis in men (due to lower asthma prevalence in men than in women). ${ }^{10}$

Given a potential protective role of testosterone against asthma and our prior negative results for asthma in a relatively small sample of US men, ${ }^{10}$ we examined the relation between serum-free testosterone and asthma, current wheeze, asthma hospitalisations and lung function in an analysis of 256419 British adults aged 40 to 69 years. 


\section{METHODS}

\section{Study design and study population}

The UK Biobank (UKB) is a large prospective population-based study established to identify determinants of complex diseases of middle and old age. Approximately 9.2 million individuals aged 40 to 69 years who lived within 22 miles of assessment centres in England, Wales and Scotland were invited to enter the cohort, and $\sim 500000(5.5 \%)$ of those individuals participated in the baseline assessment. ${ }^{11}$ Extensive data, including questionnaires, physical measures and biological samples were collected at baseline, with longitudinal follow-up for a wide range of healthrelated outcomes. ${ }^{11} 12$ Details of the methods, protocols and definitions used in the study can be found at the UKB website ( www.ukbiobank.ac.uk/). The current study was conducted using the UKB Resource under application \#43252 for a predictive model and constitutes an interim analysis. The algorithm used to select participants in this study is shown in online supplementary figure E1. Of 502524 participants recruited at baseline, 256419 (123 921 women (who did not report being pregnant) and 132498 men) had complete information on asthma status, sex hormone levels and relevant covariates, and were thus included in the current analysis of asthma. A subset of these 256419 participants, including 87137 women and 94491 men, also had measures of $\mathrm{FEV}_{1}, \mathrm{FVC}$ and $\mathrm{FEV}_{1} / \mathrm{FVC}$, and were thus included in the current analysis of lung function.

\section{Outcomes}

Asthma was defined by inclusion of 'asthma' as an answer to the following question: 'Has a doctor ever told you that you have had any of the following conditions: blood clot in the leg, blood clot in the lung, emphysema/chronic bronchitis, asthma, hay fever, allergic rhinitis or eczema?' Control subjects were participants who did not include 'asthma' as an answer to the question above. Current wheeze was defined as a 'Yes' answer to the following question: 'In the last year have you ever had wheeze or whistling in the chest?'. A hospitalisation for asthma was defined as ever having had a hospitalisation with an International Classification of Diseases Clinical Modification (ICD) code of main diagnosis compatible with asthma (ICD-9: 493.x or ICD-10: J45.x and J46.x), excluding hospitalisations with an ICD code for a main diagnosis consistent with COPD (ICD-9: J43, J44, J47 or ICD-10: 490, 491, 492, 494, 496).

Spirometry was performed using a Vitalograph Pneumotrac 6800 spirometer (Vitalograph Ltd, Buckingham, England), following European Respiratory Society/American Thoracic Society criteria for acceptability and reproducibility. ${ }^{13}$ Participants were excluded from spirometry testing if they were being treated for tuberculosis, or if they had a chest infection in the last month, history of a detached retina, a heart attack, eye surgery, surgery in the chest or abdomen in the previous 3 months or history of a collapsed lung or a pneumothorax.

\section{Serum sex hormone levels}

The UKB collected blood samples during the baseline assessment visit. ${ }^{14}$ Serum total testosterone and estradiol were measured at the UKB central laboratory by competitive binding immunoassay analysis, and sex hormone-binding globin (SHBG) was measured using a two-step sandwich immunoassay analysis on a Beckman Coulter Unicel Dxl 800 (Beckman Coulter (UK) Ltd). Internal quality control and external quality assurance schemes were used to verify assay performance. Samples with results exceeding the reportable range of the assay were diluted and reanalysed (automatic dilution). ${ }^{15}$ Values below the analytical range for estradiol (175 pmol/L) were assigned a constant $(123.7 \mathrm{pmol} / \mathrm{L})$, calculated by dividing the lower limit of detection (LLOD) by the square root of 2 (LLOD $/ \sqrt[2]{2}$ ).

Because testosterone circulates highly bound to SHBG, free testosterone was estimated using the empirical free testosterone (EFT) formula, as follows total testosterone (TT) ${ }^{16}$ :

EFT-low $(\mathrm{TT}<5 \mathrm{nM})=-6.593+19.304 \times \mathrm{TT}+0.056 \times \mathrm{SHB}-$ $\mathrm{G}-0.0959 \times \mathrm{TT} \times \mathrm{SHBG}$

EFT-high $(\mathrm{TT} \quad \geq 5 \mathrm{nM})=-52.65+24.4 \times \mathrm{TT}-0.704 \times \mathrm{SHB}-$ $\mathrm{G}-0.0782 \times \mathrm{TT} \times \mathrm{SHBG}-0.0584 \times \mathrm{TT}^{2}$

\section{Statistical analysis}

Two-sided Wald $\chi^{2}$ and Wilcoxon rank-sum tests were used for the bivariate analyses, as appropriate. Logistic regression was used for the multivariable analyses of serum-free testosterone (as quartiles) and asthma, current wheeze and at least one asthma hospitalisation, which were conducted separately in men and women. Known or potential confounders of the relation between serum-free testosterone and asthma were included in the multivariable models. All models were adjusted for age, race/ethnicity (Caucasian versus other), annual household income ( $<$ vs $\geq$ $£ 31000$ per year, near the median household income for the UK in 2019), ${ }^{17}$ body mass index (BMI), smoking status (never, former or current), pack-years of cigarette smoking, season and time of the day when the samples were collected (to account for daily and seasonal variation), serum estradiol level (see below) and (in women only) current use of oral contraceptives (OC) or hormone replacement therapy (HRT) and menopause (see below). Because most participants in the UKB were older adults, estradiol levels were below the LLOD in approximately $74 \%$ of women and $91 \%$ of men. Thus, serum estradiol was categorised as at or above versus below the LLOD for data analysis. In turn, menopause was defined by a positive answer to the following question 'have you had your menopause (periods stopped)' or (in the $15 \%$ of participants who answered 'not sure') by an age $\geq 51$ years (the average age for menopause in the UK). ${ }^{18}$ On the basis of our prior work, we tested for an interaction between serum-free testosterone level and two variables (obesity and menopause) after the final models were built.

Linear regression was used for the multivariable analysis of serum-free testosterone and lung function measures $\left(\mathrm{FEV}_{1}, \mathrm{FVC}\right.$ and $\mathrm{FEV}_{1} / \mathrm{FVC}$ ). All models were adjusted for age, race/ethnicity, annual household income, BMI, smoking status, pack-years of cigarette smoking, season and time of the day when the samples were collected, serum estradiol level, asthma status (for all participants), (in women only) current use of OC or HRT, and menopause. Models for $\mathrm{FEV}_{1}$ and $\mathrm{FVC}$ were additionally adjusted for height and height squared. Participants with extreme values for $\mathrm{FEV}_{1}$ or $\mathrm{FVC}\left(<1^{\mathrm{st}}\right.$ or $>99^{\text {th }}$ percentile) were excluded from the analysis.

Because 101876 (28\%) of the eligible participants were excluded from the analysis due to missing data for covariates (online supplementary figure E1), a multiple imputation procedure was used to include these participants in a sensitivity analysis. All statistical analyses were conducted using SAS 9.4 (SAS Institute Inc, Cary, North Carolina).

\section{Patient and public involvement}

No patients were involved in setting the research question or the outcome measures, nor were they involved in developing plans for design or implementation of the study. No patients were asked to advise on interpretation or writing up of results. 
Table 1 Main characteristics of study participants, by sex and asthma status

\begin{tabular}{|c|c|c|c|c|}
\hline \multirow[b]{2}{*}{ Characteristics } & \multicolumn{2}{|l|}{ Women $(n=123921)$} & \multicolumn{2}{|l|}{ Men $(n=132498)$} \\
\hline & Controls ( $n=109865)$ & Asthma $(n=14056)$ & Controls $(n=118251)$ & Asthma $(n=14247)$ \\
\hline Age at recruitment (years) & $55.4 \pm 8.0$ & $54.3 \pm 8.1^{*}$ & $56.4 \pm 8.1$ & $55.3 \pm 8.4^{*}$ \\
\hline Caucasian ethnicity & $104642(95.3)$ & $13301(94.6)^{*}$ & $112602(95.2)$ & $13598(95.4)$ \\
\hline Annual household income $<\mathrm{f} 31000$ & $54863(49.9)$ & $7077(50.4)$ & $52906(44.7)$ & $6172(43.2)^{*}$ \\
\hline $\mathrm{BMI}\left(\mathrm{kg} / \mathrm{m}^{2}\right)$ & $27.0 \pm 5.1$ & $28.4 \pm 6.0^{*}$ & $27.8 \pm 4.2$ & $28.0 \pm 4.4^{*}$ \\
\hline Obesity (BMI $\geq 30 \mathrm{~kg} / \mathrm{m}^{2}$ ) & $25807(23.5)$ & $4545(32.3)^{*}$ & $29624(25.1)$ & $3919(27.5)^{*}$ \\
\hline \multicolumn{5}{|l|}{ Smoking status } \\
\hline Never & $75812(69.0)$ & $9506(67.6)^{*}$ & $69392(58.7)$ & $8558(60.1)^{*}$ \\
\hline Former & $24056(21.9)$ & $3258(23.2)$ & $35436(30.0)$ & $4330(30.4)$ \\
\hline Current & $9997(9.1)$ & $1292(9.2)$ & $13423(11.4)$ & $1359(9.5)$ \\
\hline Pack-years of smoking & $6.1 \pm 12.3$ & $6.7 \pm 13.4^{*}$ & $10.6 \pm 18.2$ & $10.3 \pm 18.6$ \\
\hline Current use of $\mathrm{OC}$ or HRT & $8023(7.3)$ & $1212(8.6)^{*}$ & - & - \\
\hline Had menopause & $75474(68.7)$ & $8966(63.8)^{*}$ & - & - \\
\hline Free testosterone (pmol/L) & $11.9 \pm 8.8$ & $11.4 \pm 8.6^{*}$ & $164.9 \pm 60.1$ & $163.2 \pm 61.7^{*}$ \\
\hline Estradiol (pmol/L) & $241.4 \pm 302.7$ & $259.1 \pm 314.6^{*}$ & $132.9 \pm 0.1$ & $133.2 \pm 0.4$ \\
\hline $\mathrm{FEV}_{1}(\mathrm{~mL}) \dagger$ & $2500 \pm 489$ & $2359 \pm 506^{*}$ & $3368 \pm 660$ & $3099 \pm 726^{*}$ \\
\hline $\mathrm{FVC}(\mathrm{mL}) \dagger$ & $3266 \pm 593$ & $3179 \pm 606^{*}$ & $4457 \pm 794$ & $4331 \pm 842^{*}$ \\
\hline $\mathrm{FEV}_{1} / \mathrm{FVC}(\%) \dagger$ & $76.7 \pm 5.4$ & $74.1 \pm 6.6^{*}$ & $75.5 \pm 6.3$ & $70.9 \pm 8.2^{*}$ \\
\hline Wheeze in the last year & - & $8826(63.7)$ & - & $9341(66.5)$ \\
\hline At least one asthma hospitalisation & - & $484(3.6)$ & - & $354(2.6)$ \\
\hline
\end{tabular}

Results are shown as mean \pm SD for continuous variables, and as $\mathrm{N}(\%)$ for binary variables.

${ }^{*} \mathrm{p}<0.05$ for comparison of participants with current asthma versus controls.

†Numbers (\%) may vary due to missingness.

BMI, body mass index; HRT, hormone replacement therapy; OC, oral contraceptives.

\section{RESULTS}

Table 1 shows the main characteristics of participants by asthma status, separately in men and women. Estimates of the prevalence of physician-diagnosed asthma (heretofore called 'asthma') were $11.3 \%$ in women and $9.3 \%$ in men. Compared with women without asthma $(n=109865)$, those with asthma $(n=14056)$ were younger and less likely to be Caucasian and never smokers, to have a higher BMI and a detectable serum estradiol level, and to have a lower serum-free testosterone level and lower lung function measures. Women with asthma were also more likely to report current use of OC or HRT and to be pre-menopausal than those without asthma. Compared with men without asthma $(n=118251)$, those with asthma $(n=14247)$ were more likely to be younger and non-current smokers, and to have higher household income, lower serum-free testosterone levels and lower lung function measures.

Figure 1 shows serum-free testosterone levels by age and asthma status, separately in men and women. Serum-free testosterone levels decreased slightly with age in both men and women, with slightly higher levels among control subjects. In a multivariable analysis (figure 2 and Model 1 in online supplementary table E1), women whose serum-free testosterone level was above the first or lowest quartile (Q1) had $16 \%$ to $33 \%$ significantly lower odds of physician-diagnosed asthma than those with levels in Q1 (eg, adjusted OR (aOR) for Q4 (the fourth or highest quartile) versus $\mathrm{Q} 1=0.67,95 \% \mathrm{CI}=0.64$ to 0.71$)$. In this analysis, women who had a serum estradiol at or above the LLOD (detectable) had $8 \%$ significantly higher odds of asthma than those with a serum estradiol below the LLOD (undetectable). Moreover, the observed association between serum-free testosterone or serum estradiol and asthma was essentially unchanged after the analysis was additionally adjusted for number of live births and menstruation on the day of examination (Model 2 in online supplementary table E1) or after excluding women with missing data for the question about menopause (Model 3 in online supplementary table E1). In the multivariable analysis in men (Model 1 in online supplementary table E1 and figure 2), subjects with serum-free testosterone levels in Q4 had 13\% lower odds of asthma than those with serum-free testosterone levels in Q1 (95\% CI for $\mathrm{aOR}=0.82$ to 0.91 ). There was no significant association between serum estradiol and asthma in men.

To reduce the impact of potential misclassification of COPD as asthma, we repeated the multivariable analysis of asthma after stratification by smoking status (current smokers versus never smokers and former smokers with $<10$ pack-years of smoking). In this analysis (online supplementary table E2), a serum-free testosterone level above Q1 was significantly associated with lower odds of asthma in men and women, regardless of smoking status. A detectable serum estradiol was significantly associated with increased odds of asthma in women who were never smokers or former smokers with $<10$ pack-years of smoking, but not in current smokers. A detectable serum estradiol was not significantly associated with asthma in men, regardless of smoking status.

Table 2 shows the results of the multivariable analysis of serum-free testosterone level and current wheeze, and at least one asthma hospitalisation. In this analysis, women with asthma whose testosterone levels were in Q4 had 22\% significantly lower odds of current wheeze and $27 \%$ significantly lower odds of ever having had an asthma-related hospitalisation than women with asthma and free testosterone levels in Q1. Among men with asthma, a serum-free testosterone level in Q4 was significantly associated with $14 \%$ lower odds of current wheeze, but there was no significant association between serum-free testosterone 

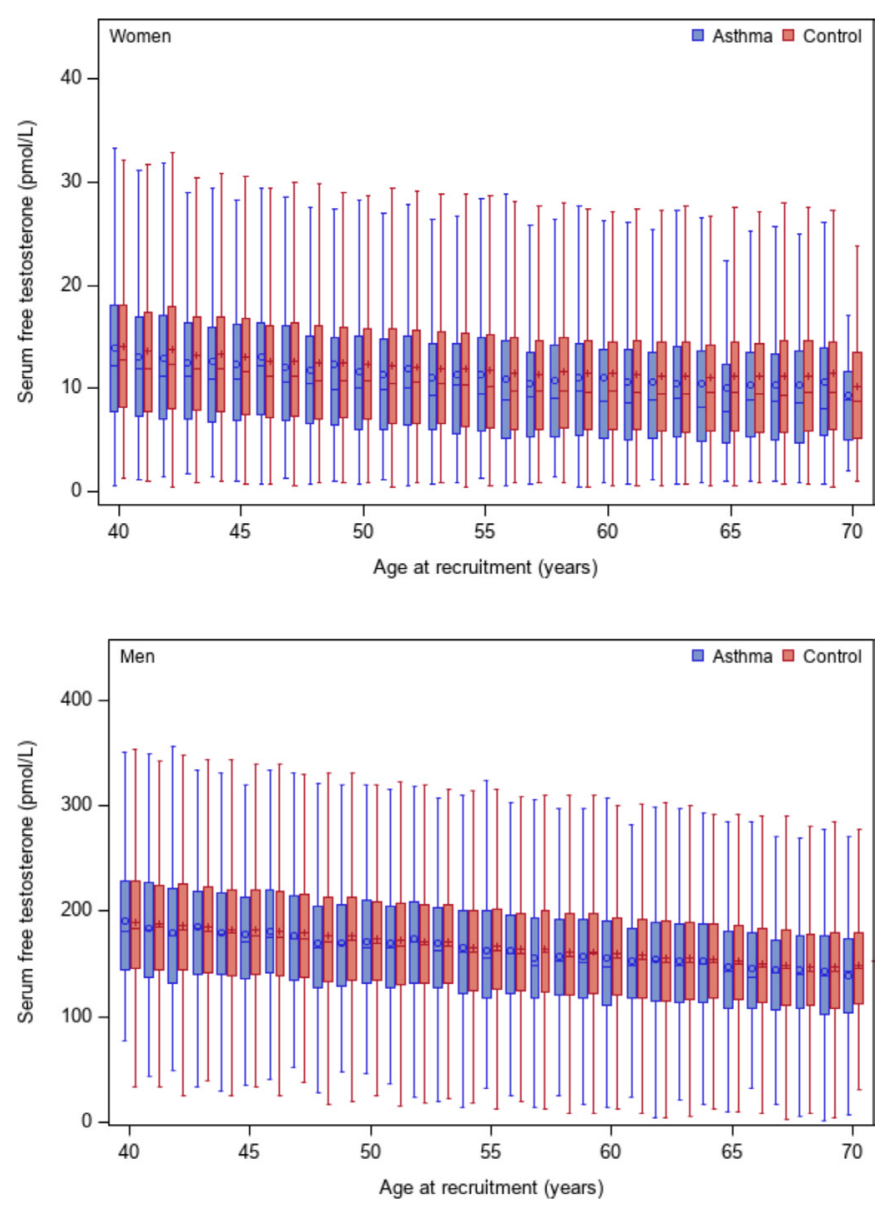

Figure 1 Serum levels of free testosterone by age and asthma status in women (upper panel) and men (lower panel).

and at least one asthma hospitalisation. A detectable serum estradiol level was not significantly associated with current wheeze or asthma hospitalisations, either in men or women.

Table 3 shows the results of the multivariable analysis of serumfree testosterone and lung function measures. Among men, each quartile increment in free testosterone was significantly
Table 2 Multivariable analysis of serum-free testosterone level and current wheeze, and at least one asthma hospitalisation

\begin{tabular}{|c|c|c|}
\hline \multirow[b]{2}{*}{ Serum levels of sex hormones } & Current wheeze & $\begin{array}{l}\text { At least one asthma } \\
\text { hospitalisation }\end{array}$ \\
\hline & \multicolumn{2}{|l|}{ OR $(95 \% \mathrm{Cl})$} \\
\hline Women with asthma $(n=14056)$ & $n=8826$ & $\mathrm{n}=484$ \\
\hline \multicolumn{3}{|l|}{ Quartiles (Q) of free testosterone, $\mathrm{pmol} / \mathrm{L}$} \\
\hline Q1 (<5.9) & 1.0 & 1.0 \\
\hline Q2 $(5.9$ to $<9.8)$ & $0.87(0.79 \text { to } 0.96)^{\dagger}$ & 0.91 (0.71 to 1.17$)$ \\
\hline Q3 (9.8 to <14.8) & $0.78(0.70 \text { to } 0.86)^{\dagger}$ & 0.86 (0.66 to 1.11$)$ \\
\hline Q4 ( $\geq 14.8)$ & $0.78(0.71 \text { to } 0.87)^{\dagger \neq}$ & $0.73(0.56 \text { to } 0.95)^{*}$ \\
\hline \multicolumn{3}{|l|}{ Estradiol, pmol/L } \\
\hline$<123.7$ & 1.0 & 1.0 \\
\hline$\geq 123.7$ & 1.10 (0.99 to 1.22 ) & 1.12 (0.86 to 1.45$)$ \\
\hline Men with asthma ( $n=14247)$ & $\mathrm{n}=9341$ & $\mathrm{n}=354$ \\
\hline \multicolumn{3}{|c|}{ Quartiles (Q) of free testosterone, pmol/L } \\
\hline Q1 $(<121.0)$ & 1.0 & 1.0 \\
\hline Q2 $(121.0$ to $<158.5)$ & 1.01 (0.91 to 1.12 ) & 0.91 (0.67 to 1.22 ) \\
\hline Q3 (158.5 to <199.2) & $0.93(0.84$ to 1.03$)$ & 0.86 (0.64 to 1.17$)$ \\
\hline Q4 ( $\geq 199.2)$ & $0.86(0.77 \text { to } 0.95)^{\dagger \neq}$ & 0.84 (0.61 to 1.15$)$ \\
\hline \multicolumn{3}{|l|}{ Estradiol, pmol/L } \\
\hline$<123.7$ & 1.0 & 1.0 \\
\hline$\geq 123.7$ & 0.96 (0.84 to 1.08$)$ & $1.20(0.85$ to 1.70$)$ \\
\hline
\end{tabular}

All models included both serum-free testosterone and serum estradiol levels, and were adjusted for age, race/ethnicity, annual household income, body mass index, smoking status, pack-years of smoking, current use of birth control pills or hormone replacement therapy and menopause status (in women), and the season and the time of the day when the examination was performed.

${ }^{*} p<0.05,{ }^{\dagger} p<0.01,{ }^{\ddagger} p$ for trend $<0.05$

associated with $26 \mathrm{~mL}$ higher $\mathrm{FEV}_{1}$ and $36 \mathrm{~mL}$ higher FVC, while having a detectable serum estradiol level was significantly associated with lower FEV 1 (by $44.7 \mathrm{~mL}$ ), lower FVC (by $50.1 \mathrm{~mL}$ ) and lower $\mathrm{FEV}_{1} / \mathrm{FVC}$ (by $0.21 \%$ ). Among women, each quartile increment in free testosterone was significantly associated with $4.3 \mathrm{~mL}$ lower FVC, while a detectable serum estradiol level was significantly associated with higher FVC (by $11.1 \mathrm{~mL}$ )

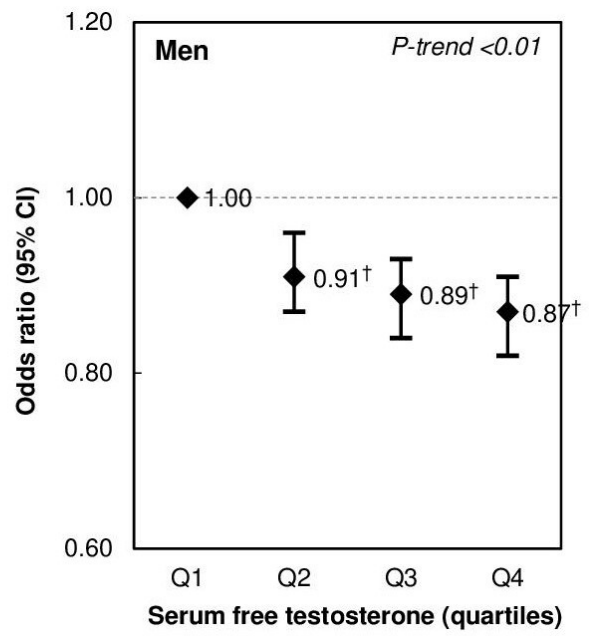

Figure 2 Multivariable analysis of serum free testosterone levels and asthma, in women (left panel) and men (right panel). All models were adjusted for age, race/ethnicity, annual household income, body mass index, smoking status, pack-years of smoking, current use of birth control pills or hormone replacement therapy and menopause status (in women), the season and the time of the day when the examination was performed and serum estradiol level. ${ }^{\dagger} p<0.01$ 
Table 3 Multivariable analysis of serum-free testosterone and lung function measures

\begin{tabular}{|c|c|c|}
\hline \multirow{2}{*}{$\begin{array}{l}\text { Serum levels of sex } \\
\text { hormones }\end{array}$} & $\begin{array}{l}\text { Women } \\
(n=87137)\end{array}$ & $\begin{array}{l}\text { Men } \\
(n=94491)\end{array}$ \\
\hline & \multicolumn{2}{|l|}{$\boldsymbol{\beta}(95 \% \mathrm{Cl})$} \\
\hline \multicolumn{3}{|c|}{ Free testosterone (pmol/L), per quartile increment } \\
\hline $\mathrm{FEV}_{1}(\mathrm{~mL})$ & $-2.3(-4.6$ to 0.3$)$ & $26.0(22.8 \text { to } 29.2)^{*}$ \\
\hline $\mathrm{FVC}(\mathrm{mL})$ & $-4.3(-7.1 \text { to }-1.5)^{*}$ & $36.0(32.3 \text { to } 39.8)^{*}$ \\
\hline $\mathrm{FEV}_{1} / \mathrm{FVC}(\%)$ & $0.03(-0.01$ to 0.06$)$ & $-0.004(-0.04$ to 0.04$)$ \\
\hline \multicolumn{3}{|l|}{ Estradiol $\geq 123.7 \mathrm{pmol} / \mathrm{L}$} \\
\hline $\mathrm{FEV}_{1}(\mathrm{~mL})$ & $5.40(-2.15$ to 12.94$)$ & $\begin{array}{l}-44.70(-56.52 \text { to } \\
-38.88) \dagger\end{array}$ \\
\hline $\mathrm{FVC}(\mathrm{mL})$ & $11.13(1.99 \text { to } 20.28)^{*}$ & $\begin{array}{l}-50.11(-63.94 \text { to } \\
-36.28) \dagger\end{array}$ \\
\hline $\mathrm{FEV}_{1} / \mathrm{FVC}(\%)$ & $-0.12(-0.23 \text { to }-0.02)^{*}$ & $-0.21(-0.34$ to -0.07$) \dagger$ \\
\hline
\end{tabular}

All models included both serum-free testosterone and serum estradiol, and were adjusted for age, race/ethnicity, annual household income, body mass index, asthma status, smoking status, pack-years of cigarette smoking, current use of birth control pills or hormone replacement therapy and menopause status (in women), and the season and the time of the day when the examination was performed. Models for $\mathrm{FEV}_{1}$ and FVC were additionally adjusted for height and height squared. ${ }^{*} \mathrm{p}<0.05$. $\mathrm{tp}<0.01$.

and lower $\mathrm{FEV}_{1} / \mathrm{FVC}$ (by $0.12 \%$ ). We obtained similar results in a secondary analysis of lung function in which z-scores were calculated for lung function measures, based on the Global Lung Function Initiative $2012^{19}$ (online supplementary table E3).

Because of our prior findings in a US cohort of adults aged 18 to 79 years, we tested for an interaction between obesity (a BMI $\left.\geq 30 \mathrm{~kg} / \mathrm{m}^{2}\right)$ and serum-free testosterone on asthma. This interaction was not significant in women $(\mathrm{p}=0.53)$ or men $(\mathrm{p}=0.10)$. Among women, we also tested for an interaction between menopause and serum-free testosterone on asthma, finding no significant interaction $(\mathrm{p}=0.37)$.

After imputing data for missing covariates, we repeated the multivariable analysis of serum-free testosterone and asthma in all eligible participants $(\mathrm{n}=358295)$. This sensitivity analysis yielded similar results to those from the analysis that excluded participants with missing covariates (online supplementary table E4).

\section{DISCUSSION}

In a large population-based study of British adults, elevated serum levels of free testosterone were significantly associated with lower odds of physician-diagnosed asthma and current wheeze in middle-aged and older women and men. Moreover, an elevated free testosterone level was associated with decreased odds of at least one asthma hospitalisation in women, and with higher $\mathrm{FEV}_{1}$ and $\mathrm{FVC}$ in men.

Few epidemiological studies have examined free testosterone and asthma in adults. In our previous analysis of adults (ages 18 to 79 years) who participated in the US National Health and Nutrition Examination Survey (NHANES), we reported that higher levels of serum-free testosterone were associated with lower odds of current asthma in women but not in men. ${ }^{10}$ Our finding of a significant inverse association between a high serumfree testosterone level and asthma among men in the current analysis is thus novel and likely due to greater statistical power, due to a much larger sample size in the UKB than in NHANES. ${ }^{10}$ Alternatively, these results and our finding of no interaction between obesity and free testosterone in women with asthma in the UKB cohort could be explained by differences in the age range of participants in NHANES versus those in the UKB. Consistent with our current results, a human phase II clinical trial reported that nebulised dehydroepiandrosterone-3-sulphate (DHEAS) improved asthma control and symptoms in adults with poorly controlled moderate-to-severe asthma on inhaled corticosteroids and long-acting $\beta_{2}$-agonists. ${ }^{20}$ Moreover, a study of 450 US men aged 40 to 63 years with COPD showed that subjects on testosterone replacement therapy had a $4.2 \%$ to $9.1 \%$ reduction in hospitalisations compared with those on no such therapy. ${ }^{21}$

Testosterone may protect against asthma through systemic and airway-specific anti-inflammatory effects. In a murine model, testosterone was shown to decrease dust mite-induced eosinophilic and neutrophilic inflammation in the lungs, partially through androgen receptor (AR) signalling. ${ }^{22}$ In another murine model, testosterone was shown to decrease Alternaria-extractinduced interleukin (IL)-5, IL-13 and lung eosinophils by attenuating group two innate lymphoid cells (ILC2). ${ }^{23}$ Other studies have shown that androgens can induce airway smooth muscle relaxation. In guinea pig airways, testosterone at physiological concentrations reduced reactivity of smooth muscle by diminishing intracellular calcium $\left(\mathrm{Ca}^{2+}\right) \mathrm{i}$ increment through modifying $\mathrm{IP}_{3}$ receptor (ITPR). ${ }^{24}$ Androgens have also been shown to weaken tumour necrosis factor (TNF)- $\alpha$ or IL-13-induced enhancement of $\left(\mathrm{Ca}^{2+}\right) \mathrm{i}$ in human airway smooth muscle cells, which lessens airway responsiveness. ${ }^{25}$ Collectively, bronchodilating and anti-inflammatory effects of testosterone may explain our results for asthma, current wheeze and asthma hospitalisations.

Little is known about the effects of testosterone on lung function in adult women. In a study of 1768 community-dwelling adult men (of whom 14\% reported asthma and $2.7 \%$ reported COPD), total testosterone and DHT were positively associated with FEV and FVC. ${ }^{26}$ Among 2197 men with an average age of 66 years (of whom $4.7 \%$ reported COPD), higher total and free testosterone levels were significantly associated with increased $\mathrm{FEV}_{1}$ and FVC. ${ }^{27}$ In a study of boys with current asthma (aged 6 to 18 years), DHEAS was positively associated with FEV and FVC, and improved asthma control. ${ }^{28}$ Our results are thus consistent with those of prior studies in men, but this is (to our knowledge) the first study to examine free testosterone and lung function in women. The observed weak negative effect of free testosterone on FVC (but not on $\mathrm{FEV}_{1}$ or $\mathrm{FEV}_{1} / \mathrm{FVC}$ ) in women could be explained by residual confounding due to unmeasured factors affecting hormonal variations (eg, type/length/dose of hormonal contraception or replacement therapy, or follicular phase of the menstrual cycle).

The discrepant direction and magnitude of the estimated effects of testosterone on lung function between women and men are intriguing. In general, adult men have higher $\mathrm{FEV}_{1}$ and FVC than adult women (online supplementary figure E2), which may explain the larger effect size of free testosterone on lung function in men. Moreover, unmeasured androgens (eg, DHT or DHNES) or ARs may partly explain these findings. In mouse models, DHT downregulated Th2 inflammation in the lung, but ARs enhanced such inflammation by upregulating M2 macrophage polarisation that induces eosinophil recruitment. ${ }^{29}$

In our previous analysis in NHANES, in which there were both a LLOD and a broader range of serum estradiol levels than those in the current study, ${ }^{10}$ we found no significant association between serum estradiol and asthma in our main analysis. Although our current finding of an association between a detectable serum estradiol and asthma and reduced $\mathrm{FEV}_{1} / \mathrm{FVC}$ in women, and reduced lung function measures $\left(\mathrm{FEV}_{1}, \mathrm{FVC}\right.$ 
and $\mathrm{FEV}_{1} / \mathrm{FVC}$ ) in men may be due to true enhancement of allergic airway inflammation, ${ }^{7}$ these results must be cautiously interpreted because of the high proportion of participants with undetectable estradiol levels and lack of a significant association between a detectable serum estradiol and current wheeze or asthma hospitalisations in participating men and women.

We acknowledge additional study limitations. First, we cannot examine temporal relationships in this cross-sectional study. Second, serum progesterone was not measured in the UKB, and thus we could not examine whether progesterone affects asthma or lung function. Third, a 'healthy volunteer' selection bias has been suggested for the UK Biobank study. Compared with the general population, participants in the UKB were less likely to be obese or to smoke, and had fewer self-reported health conditions. ${ }^{30}$ Thus, our findings may not be generalisable to the British population at large. Fourth, recall bias and misclassification of asthma are possible, as this disease was defined using a combination of self-reported (ever) asthma (which the participant had to select from a list including other chronic conditions) and self-reported (ever) physician-diagnosed asthma. However, we obtained similar results for current wheeze, and our findings for asthma were unchanged in an analysis of data from never smokers or former smokers with $<10$ pack-years of smoking. Finally, we lack data on several potential confounders, including allergic sensitisation, insulin resistance and environmental exposure to endocrine disruptors.

In summary, we found that elevated serum levels of free testosterone were significantly associated with reduced odds of asthma and current wheeze in middle-aged and older British men and women. In these adults, we also show that elevated free testosterone levels were associated with decreased odds of at least one asthma-related hospitalisation in women, as well as higher $\mathrm{FEV}_{1}$ and FVC in men. Taken together with prior results from experimental and observational studies, our findings suggest that free testosterone contributes to the pathogenesis of asthma in adults.

Acknowledgements We thank the UK Biobank participants. This research was conducted using the UK Biobank Resource (application \# 43252).

Contributors $\mathrm{Y}-\mathrm{YH}, \mathrm{EF}$ and JCC participated in study design, implementation and data analysis. QY, GY and WC participated in data management and data analysis. $\mathrm{Y}-\mathrm{YH}, \mathrm{EF}$ and JCC wrote the first draft of the manuscript. All authors reviewed and approved the final version of the submitted manuscript. JCC had full access to all the study data and takes responsibility for the integrity of the data and the accuracy of the data analysis.

Funding Y-YH's contribution was supported by grant MD011764 from the US National Institutes of Health (NIH). JCC's contribution was supported by grants HL117191, HL119952 and MD011764 from the US NIH. EF's contribution was supported by grants HL149693 from the US NIH. QY's contribution was supported by grant HL138098 from the US NIH. This study was conducted using the UK Biobank Resource under Application Number 43252.

Competing interests JCC has received research materials from Merck and GSK (inhaled steroids), and Pharmavite (vitamin D and placebo capsules), to provide medications free of cost to participants in $\mathrm{NIH}$-funded studies, unrelated to the current work.

Patient consent for publication Not required.

Ethics approval The UKB was approved by the UK National Health Service National Research Ethics Service (Ref 11/NW/0382) and informed consent was obtained from all participants.

Provenance and peer review Not commissioned; externally peer reviewed.

Data availability statement Data may be obtained from a third party and are not publicly available. The UK Biobank data are available from the UK Biobank upon request (www.ukbiobank.ac.uk/).

ORCID iD

Erick Forno http://orcid.org/0000-0001-6497-9885

\section{REFERENCES}

1 Global Asthma Network. The global asthma report 2018. Auckland, New Zealand.

2 Mukherjee M, Stoddart A, Gupta RP, et al. The epidemiology, healthcare and societal burden and costs of asthma in the UK and its member nations: analyses of standalone and linked national databases. BMC Med 2016;14:113.

3 Asthma UK. Asthma facts and statistics London, England, 2020. Available: https:// www.asthma.org.uk/about/media/facts-and-statistics/ [Accessed 5 Feb 2020].

4 Almqvist C, Worm M, Leynaert B, et al. Impact of gender on asthma in childhood and adolescence: a GA2LEN review. Allergy 2008;63:47-57.

5 Sathish V, Martin YN, Prakash YS. Sex steroid signaling: implications for lung diseases. Pharmacol Ther 2015;150:94-108.

6 McCleary N, Nwaru BI, Nurmatov UB, et al. Endogenous and exogenous sex steroid hormones in asthma and allergy in females: A systematic review and meta-analysis. J Allergy Clin Immunol 2018;141:1510-3.

7 Fuseini H, Newcomb DC. Mechanisms driving gender differences in asthma. Curr Allergy Asthma Rep 2017;17:19.

8 Shah R, Newcomb DC. Sex bias in asthma prevalence and pathogenesis. Front Immunol 2018;9:2997.

9 Yung JA, Fuseini H, Newcomb DC. Hormones, sex, and asthma. Ann Allergy Asthma Immunol 2018;120:488-94.

10 Han Y-Y, Forno E, Celedón JC. Sex steroid hormones and asthma in a nationwide study of U.S. adults. Am J Respir Crit Care Med 2020;201:158-66.

11 Sudlow C, Gallacher J, Allen N, et al. Uk Biobank: an open access resource for identifying the causes of a wide range of complex diseases of middle and old age. PLoS Med 2015;12:e1001779.

12 UK Biobank. Key documents for a large-scale prospective epidemiological resource. Available: http://www.ukbiobank.ac.uk/key-documents/ [Accessed 4 Feb 2020].

13 Miller MR, Hankinson J, Brusasco V, et al. Standardisation of spirometry. Eur Respir J 2005;26:319-38.

14 Elliott P, Peakman TC, Biobank UK, UK Biobank. The UK Biobank sample handling and storage protocol for the collection, processing and archiving of human blood and urine. Int J Epidemiol 2008:37:234-44.

15 UK Biobank. UK Biobank Biomarker Project - Companion Document to Accompany Serum Biomarker Data, 2019. Available: http://biobank.ndph.ox.ac.uk/showcase/ showcase/docs/serum_biochemistry.pdf [Accessed 4 Feb 2020].

16 Ly LP, Handelsman DJ. Empirical estimation of free testosterone from testosterone and sex hormone-binding globulin immunoassays. Eur J Endocrinol 2005;152:471-8.

17 Office for National Statistics. Average household income, UK: financial year ending 2019. London, England, 2020.

18 National Health Service. Overview -Menopause United Kingdom, 2018. Available: https://www.nhs.uk/conditions/menopause/ [Accessed 10 Mar 2020].

19 Quanjer PH, Stanojevic S, Cole TJ, et al. Multi-Ethnic reference values for spirometry for the 3-95-yr age range: the global lung function 2012 equations. Eur Respir J 2012;40:1324-43.

20 Wenzel SE, Robinson CB, Leonard JM, et al. Nebulized dehydroepiandrosterone3-sulfate improves asthma control in the moderate-to-severe asthma results of a 6-week, randomized, double-blind, placebo-controlled study. Allergy Asthma Proc 2010:31:461-71.

21 Baillargeon J, Urban RJ, Zhang W, et al. Testosterone replacement therapy and hospitalization rates in men with COPD. Chron Respir Dis 2019;16:1479972318793004

22 Fuseini H, Yung JA, Cephus JY, et al. Testosterone decreases house dust mite-induced type 2 and IL-17A-Mediated airway inflammation. J Immunol 2018;201:1843-54.

23 Cephus J-Y, Stier MT, Fuseini H, et al. Testosterone attenuates group 2 innate lymphoid cell-mediated airway inflammation. Cell Rep 2017;21:2487-99.

24 Montaño LM, Flores-Soto E, Reyes-García J, et al. Testosterone induces hyporesponsiveness by interfering with $\mathrm{IP}_{3}$ receptors in guinea pig airway smooth muscle. Mol Cell Endocrinol 2018;473:17-30.

25 Kalidhindi RSR, Katragadda R, Beauchamp KL, et al. Androgen receptor-mediated regulation of intracellular calcium in human airway smooth muscle cells. Cell Physiol Biochem 2019:53:215-28.

26 Mohan SS, Knuiman MW, Divitini ML, et al. Higher serum testosterone and dihydrotestosterone, but not oestradiol, are independently associated with favourable indices of lung function in community-dwelling men. Clin Endocrinol 2015:83:268-76

27 Svartberg J, Schirmer $\mathrm{H}$, Medbø A, et al. Reduced pulmonary function is associated with lower levels of endogenous total and free testosterone. The Tromsø study. Eur J Epidemiol 2007:22:107-12.

28 DeBoer MD, Phillips BR, Mauger DT, et al. Effects of endogenous sex hormones on lung function and symptom control in adolescents with asthma. BMC Pulm Med 2018; 18:58.

29 Becerra-Díaz M, Strickland AB, Keselman A, et al. Androgen and androgen receptor as enhancers of M2 macrophage polarization in allergic lung inflammation. J Immunol 2018:201:2923-33

30 Fry A, Littlejohns TJ, Sudlow C, et al. Comparison of sociodemographic and health-related characteristics of UK Biobank participants with those of the general population. Am J Epidemiol 2017;186:1026-34. 